\title{
SEGMENTATION OF LUNG LOBES AND NODULES IN CT IMAGES
}

\author{
Anitha. $S^{1}$ and Sridhar. $S^{2}$ \\ ${ }^{1}$ Department of Computer Science and Engineering, College of Engineering, Guindy \\ any_kanny@yahoo.co.in \\ ${ }^{2}$ Department of Computer Science and Engineering, College of Engineering, Guindy \\ sridhar@es.annauniv.edu
}

\begin{abstract}
The objective of this paper is to develop a segmentation system in order to assist the surgeons to remove the portion of lung for the treatment of certain illness such as lung cancer, and tumours. The fissures of lung lobes are not seen by naked eyes in low dose CT image, there is a proposal for automatic segmentation system. The lung lobes and nodules in CT image are segmented using two stage approaches such as modified adaptive fissure sweep and adaptive thresholding. Initially pre-processing is used to remove the noise present in CT image using filter, then the fissure regions are located using adaptive fissure sweep technique, then histogram equalization and region growing is applied to refine the oblique fissure. Lung Nodules are segmented using thresholding. The comparative analysis of manual and automatic segmentation for fissure verification has been performed statistically. The analysis is made on 20 set of images.
\end{abstract}

\section{KEYWORDS}

CT, Fissure Sweeping, Lung Segmentation, Lung Nodules.

\section{INTRODUCTION}

Multisided CT imaging is the primary digital technique for imaging the lung for the detection of pulmonary (lung) disease such as lung cancer, tumour, and cystic fibrosis. The clinical CT image of the lung is shown in Figure 1 and its general anatomy is shown in Figure 2. In order to segment lung lobes, fissures or boundaries are detected based on apriori knowledge from the anatomic atlas, this method uses ridge operator and graph search [22]. By using 3D watershed transform lung lobes are segmented, in this method distance map is performed on vessel mask and then combine the original, refine the fissures using interactive watershed transform [11]. The first step for lung lobe segmentation is lung segmentation, in this method lung regions are extracted by using threshold and left and right lungs are segmented using dynamic programming [6]. Fissure extraction using Vander burg's linear feature detector and morphological operators for segmenting lung lobes from $1 \mathrm{~mm}$ clinical CT image [12], $0.2 \mathrm{~mm}$ isotropic CT images [10]. Fissure near the lesions are calculated using surface curvature analysis [9]. Boundary of an object is identified using spline contours and iterative energy minimization formula, however this method fails when large gaps are present [2] [3] [16]. Fissures are present in the lung where there are no vascular or bronchial trees and hence it is necessary to segment the vascular and the bronchial trees by considering the grey level information, 3D shape constraint, and apriori knowledge [5]. Lung lobes are segmented using apriori knowledge [23] and 3D shape constraint [18]. In this method fissures are identified using graph search and fuzzy reasoning. By using the Gaussian, sobel and ridge operators fissures are detected using shape based curve growing method in $1.25 \mathrm{~mm}$ CT image [19] [15]. The pre requisite for the fissure detection to segment the lung lobes is lung segmentation, in this paper segmentation by registration is used [20]. Lung lobes are segmented in clinical CT image using adaptive fissure sweeping for finding the fissure region and using watershed transform to enhance the fissure [17]. Pulmonary fissures are segmented by extracting the vascular 
trees [8]. 3D visualization of lung cavities is important for surgical planning of treating lung disease [7] [1]. Methods such as variation approach [21], graph cuts [4], guided random walks [14] are used for finding the object boundaries, however this method requires manual intervention and high computation. In order to identify the actual fissure locations and curvatures from the fissure regions using discrete wavelet transform [5]. Fissure regions are identified using fissure sweeping technique and then the locations are identified using discrete wavelet transform [24].

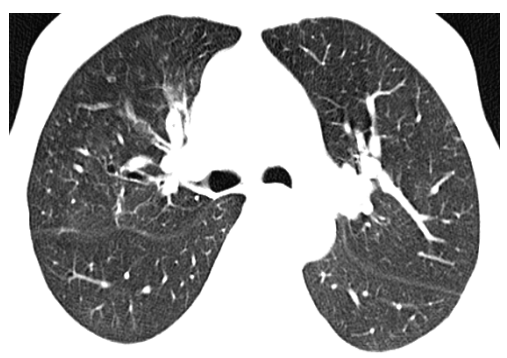

Figure1. Clinical CT Image

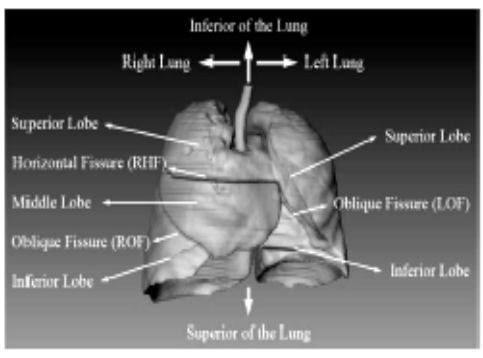

Figure2. General anatomy of Human lung

\section{Proposed TeChNiQue}

The proposed technique mainly consists of four subdivisions:

2.1 Image Enhancement and Segmentation.

2.2 Fissure Detection for identifying the fissure regions.

2.3 Fissure Refinement using region growing method.

2.4 Nodule Segmentation using Adaptive Threshold

The system architecture is presented in Figure 3.

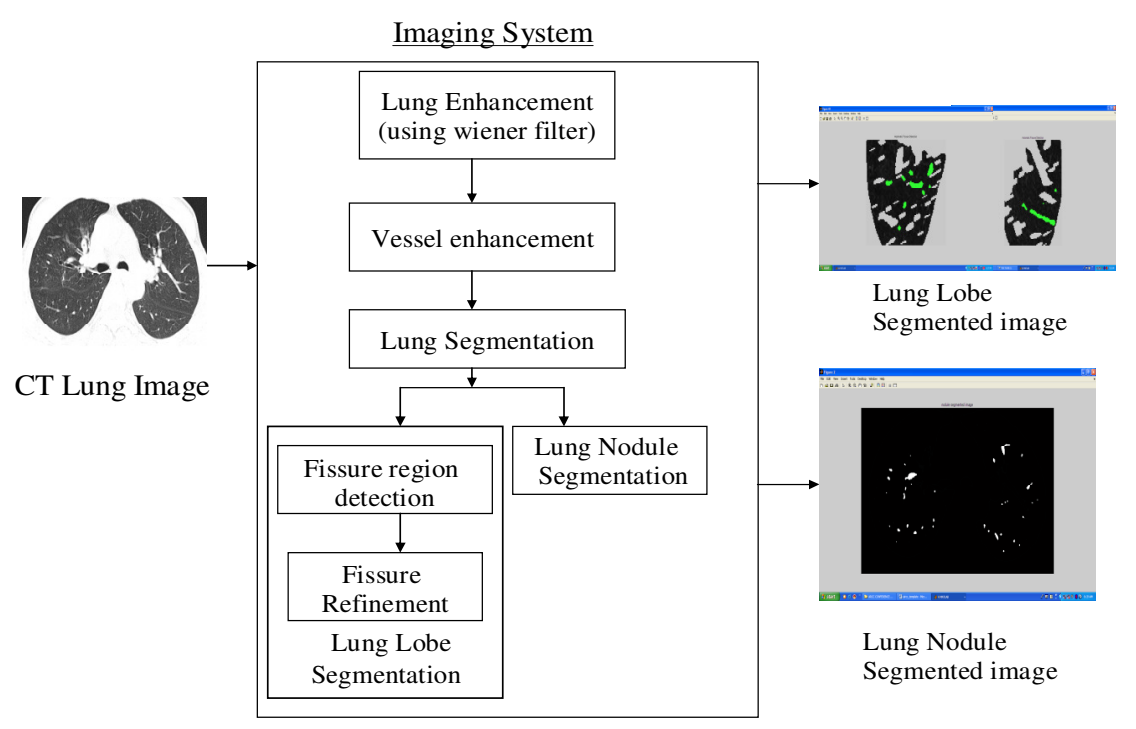

Figure 3.Block Diagram 


\subsection{Image Enhancement and Segmentation}

Pre-processing is done to remove the noise present in the CT image. In order to remove the Gaussian noise, Wiener filter is used. Since Wiener filter gives an estimate of the original uncorrupted image with minimum mean square error, and hence Wiener filter is used for removing the noise present in the lung CT image. Bronchial and the vascular trees are enhanced in the CT image using the morphological Hat operation. The morphological operations are based on simple mathematical concepts from set theory. These operators are particularly useful for the analysis of binary images and common usage include edge detection, noise removal and image enhancement. Segmentation refers to the process of partitioning a digital image into multiple segments. The goal of segmentation is to simplify and/or change the representation of an image into something that is more meaningful and easier to analyse. Image segmentation is typically used to locate objects and boundaries (lines, curves, etc.) in images. More precisely, image segmentation is the process of assigning a label to every pixel in an image such that pixels with the same label share certain visual characteristics. In this paper adaptive threshold method is used to segment the left and right lungs.

\subsubsection{Steps for lung enhancement}

Pre-processing of image is done using wiener filter. Wiener filter of size $3 / 3$ pixel is optimum. Resulting image contains less speckle noise. Vascular and Bronchial trees are enhanced using the morphological operators such as the bottom and top hat filters.

\subsubsection{Steps for lung Segmentation}

Left and right lung are segmented using the adaptive thresholding. Lung is segmented using thresholding and connected component labelling. Mean of the CT image is used as the threshold value.

\subsection{Fissure Detection for identifying the fissure regions}

The fissure sweeping method is used for finding the fissure region, in fissure sweeping predefined fissure angles are used to find the fissures. Fissure angle is defined as the angle between the fissure and the flat horizontal line. For finding the fissures in the superior region of the lungs positive fissure angle is used and to detect the fissure in the inferior region of the lung negative fissure angle is used.

\subsubsection{Steps for Fissure Region Sweeping}

It uses predefined fissure angle, fissure angle is the angle between the fissure and flat horizontal line. At each angle the technique scans the CT image to detect the empty region where fissure could be located. Euclidean distances are used to detect the search region.

$\mathrm{D}_{\mathrm{E}}=\mathrm{D}_{\mathrm{BW}}-\mathrm{D}_{\mathrm{W}}$

Where $\mathrm{D}_{\mathrm{E}}=$ Distance between the boundary and the centre point of the empty region.

$\mathrm{D}_{\mathrm{BW}}=$ Distance between the boundary and the centre point of the reference region.

$\mathrm{D}_{\mathrm{W}}=$ Distance between the boundary and centre point of the non empty region.

\subsection{Fissure Refinement}

Fissure locations and curvatures are identified within a fissure region. The contrast of the lobar fissure is poor, histogram equalizer is used to increase the contrast and then fissure are refined using region growing method. The region growing method determines the empty regions between vascular and bronchial trees of lung lobes. The centre of empty region provides fissure's location. 


\subsubsection{Steps for Fissure Refinement}

Fissure locations are identified using region growing method. Initially the contrast is enhanced using histogram equalization. Region growing approach is used to detect the fissure. In region growing method fissure's centre line is used as the seed point. The seed grows in an orthogonal direction to that of the centre line of the fissure region found from the fissure sweeping technique.

\subsection{Nodule Segmentation}

Nodule is a mass of tissue located in the lung. It appears as round, white shadows on CT image. Mass of $25 \mathrm{~mm}$ or larger can cause cancer. Therefore it is necessary to segment lung nodules from blood vessel at an earlier stage. Thresholding is used to segment lung nodules, since it takes less time and provides an efficient method to segment nodules.

\subsubsection{Steps for Nodule Segmentation}

Thresholding method is used to automatically detect the nodules present in lung. Mean pixel intensity of the image is taken as the threshold.

\section{IMPLEMENTATION}

\subsection{Pre-processing for the removal of noise}

The CT Images normally contains artefacts, noise which will not be suitable for further processing and hence it has to be pre-processed to reduce the noise using Wiener filter. Wiener filter is used because of following advantages.

\section{Advantages of Wiener filter}

i. It takes very short time to find the optimal solution as the apriori knowledge about the image is taken into consideration.

ii. It controls the output error as it is optimal.

iii. It is straightforward to design and it is faster.

iv. It is a least mean square filter.

The disadvantages of Wiener filter is that the results are too blurred and it is spatially invariant, but these are not observed in the experimentation result and hence Wiener filter works better when compared with respect to other. The main constraint in the use of Wiener filtering is that signal and noise should be Gaussian processes for optimality. The noise in the image can be modelled as Gaussian. Gaussian is the natural way of modelling noise. Therefore Wiener filter is used for removal of noise in CT image. Wiener filer gives an estimate of the original uncorrupted image with minimum mean square error and the estimate is the non linear function of the corrupted image. Wiener filters of size $3 * 3$ are used to remove the e noise present in the CT image, two of the error metrics are used to evaluate the filter with mean square error (MSE) and the peak signal to noise ratio (PSNR). The mean square error is the cumulative squared error between the compressed and the original image, whereas peak signal to noise ratio is a measure of the peak error. The mathematical formulae for the two are

MSE $=1 / \mathrm{MN}\left(\mathrm{I}(\mathrm{x}, \mathrm{y})-\mathrm{I}^{\prime}(\mathrm{x}, \mathrm{y})\right)----(1)$

PSNR $=20 * \log 10(255 /$ sqrt(MSE) $)----(2)$

Where $I(x, y)$ is the original image, $I^{\prime}(x, y)$ i the approximated version (which is actually the decompressed image) and M,N are the dimensions of the images. A lower value for MSE means lesser error, and as seen from the inverse relation between the MSE and PSNR, this translates to a high value of PSNR. Logically, a higher value of PSNR is good because it means that the ratio of

Signal to Noise is higher. Here, the 'signal' is the original image, and the 'noise' is the error in reconstruction. According to equation 2 PSNR is calculated in Table 1 for the CT images. 


\begin{tabular}{|c|c|}
\hline Patient \# & PSNR (db) \\
\hline 1 & 42.1595 \\
\hline 2 & 42.884 \\
\hline 3 & 37.0637 \\
\hline 4 & 37.1547 \\
\hline
\end{tabular}

Table1. Peak signal to noise ratio for the CT image

According to the equation (1) and (2) the value of mean square error and the peak signal to noise ratio is given for the Figure 4 as 42.1595

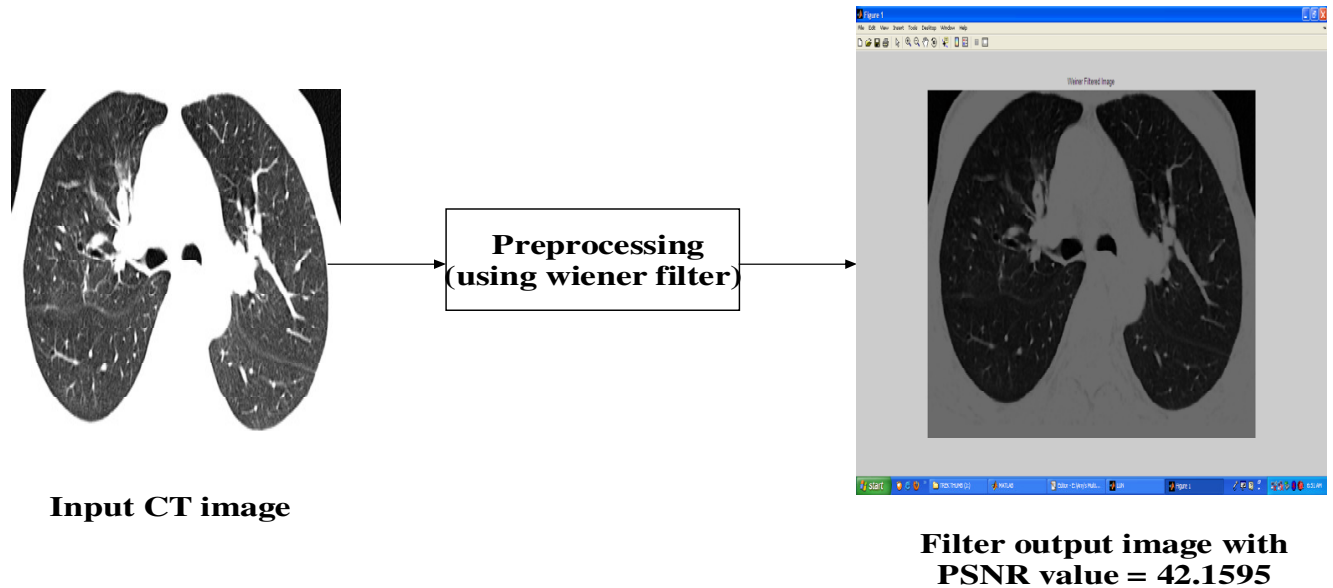

Figure 4. Wiener Filtered Image

\subsection{Vessel Enhancement}

Vascular and Bronchial tress are enhanced using the morphological operations. Morphology is a technique of image processing based on shapes. The value of each pixel in the output image is based on a comparison of the corresponding pixel in the input image with its neighbours. The first step is to create structure element. Morphological operations like top hat and bottom hat filters are used. Top-hat filtering is used to correct uneven illumination when the background is dark. Top hat operation contains peaks of object that fit the structuring element. Bottom hat operations are used to fill the gap between the objects.

Vessel Enhanced = Input image +top hat (Input image) - bottom hat (Input image) ----- (3). The output is obtained from equation (3), which is given the Figure 5. 


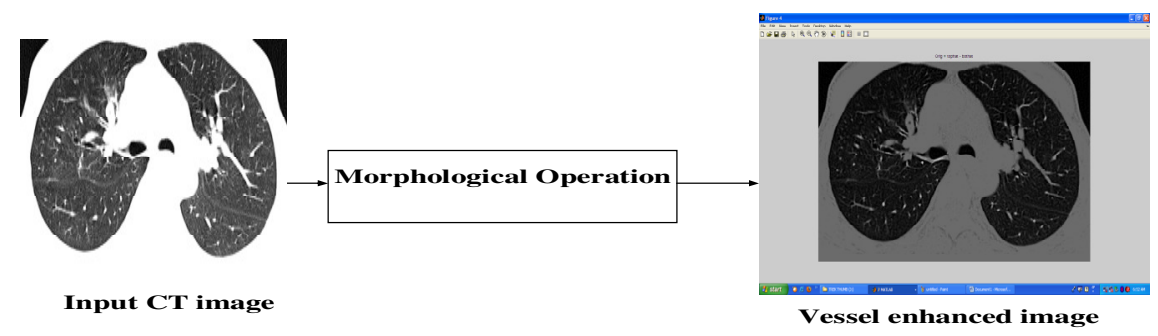

Figure 5.Vessel Enhanced Image

\subsection{Lung Segmentation}

Here the left and the right lung are segmented using adaptive thresholding. Threshold values are selected based on the mean value of the CT image. The output of lung segmentation for CT images is shown in Figure 6

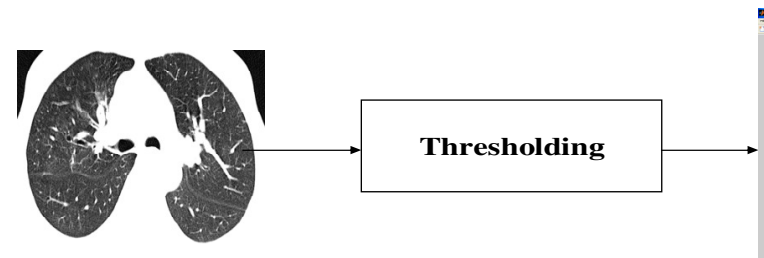

Input CT image

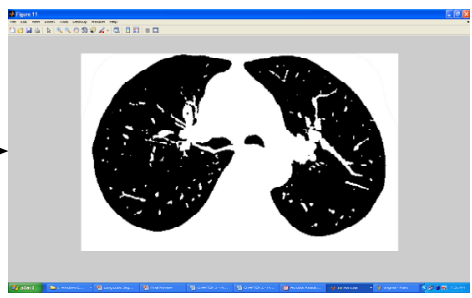

Lung segmented image

Figure 6.Lung Segmented Image

\subsection{Fissure Region detection}

Fissure regions are identified using the fissure sweeping technique and the fissures are enhanced using region growing method. The fissure sweep technique finds the fissure regions in the preprocessed CT images. The fissure sweep technique finds the fissure regions in the pre-processed CT images. The general anatomic structure of the lung is shown in Figure1, in which the lobar fissures separate the lung lobes where no major vascular or bronchial trees cross. This signifies that in the pre-processed binary images, fissures are represented by large amounts of air or space extending from the middle to the lateral side of the lungs. The fissure sweep technique uses this knowledge to coarsely define void regions where fissures could be present. The fissure sweep 
technique first calculates a set of predefined fissure angles. It is defined by angles between the fissures and a flat horizontal line using knowledge from the general lung anatomy.

\section{ALGORITHM}

Input: Threshold image

Output: Fissure detected image

\section{STEPS}

1. Take the input image.

2. Draw a horizontal reference line and the fissure line

3. Keep the horizontal line is fixed and varies fissure line from the top to bottom which scans the input image

4. Determine the void region by checking the continuous number of black pixel from the scanning process.

5. The algorithm for fissure sweeping is implemented in the Figure 7 and the fissure swept result is shown in the Figure 8.

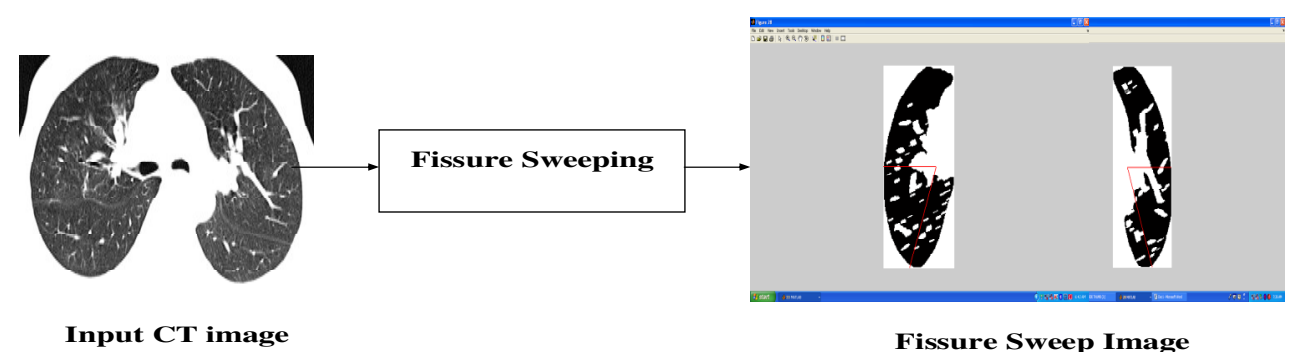

Figure 7. Fissure Sweeping

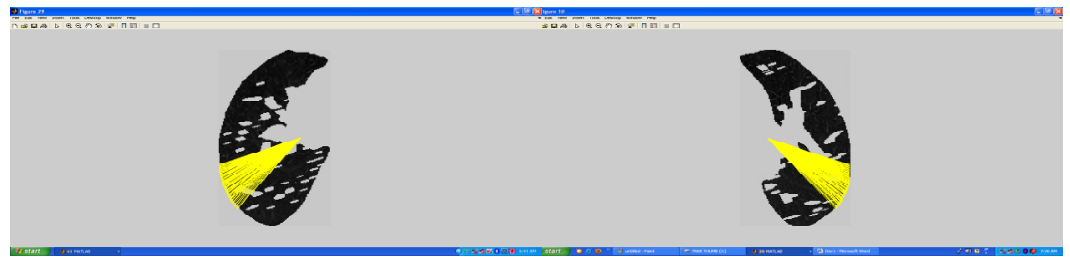

Figure 8.Fissure Swept Result 


\subsection{Fissure Refinement}

Fissures are enhanced in the lung CT image using Region growing method, initially histogram equalization is used to enhance the contrast of the fissure region, and then the Region growing method is applied to refine the fissure. Fissures are generally situated between the vascular and the bronchial trees of the two adjacent lung lobes. In Region growing method it finds the empty regions between the vascular and bronchial trees of the two lung lobes. The centre of these empty regions gives approximate fissure locations. In conventional region growing technique the seed point goes in all directions whereas in this region growing technique the centre of the fissure region is used as the seed point. This prevents searching the fissure from deviating too far from the fissure angle. The seed grows in a direction outwards is perpendicular to the middle of the fissure region and the output of fissure refined image is shown in Figure 9.

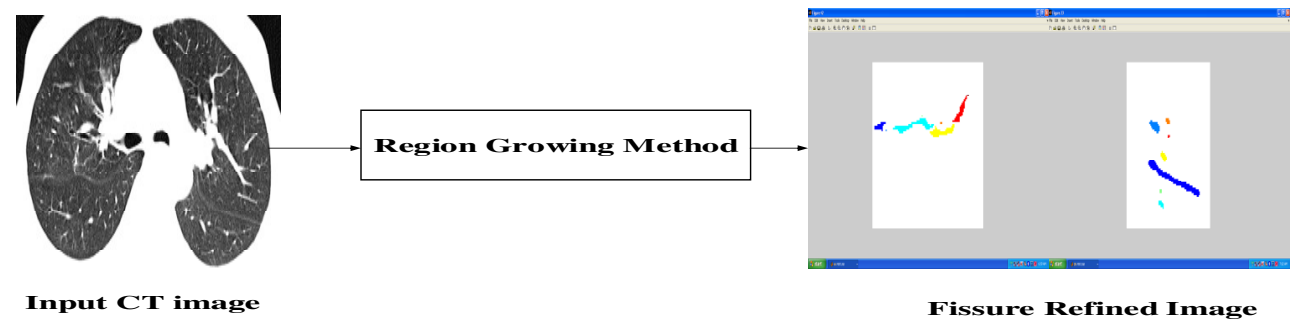

Figure 9.Fissure refined image

\subsection{Lung Nodule Region Segmentation}

Lung Nodules are segmented using adaptive thresholding. Mean of CT image is used as threshold. The Lung Nodule Segmented Image is shown in Figure10.

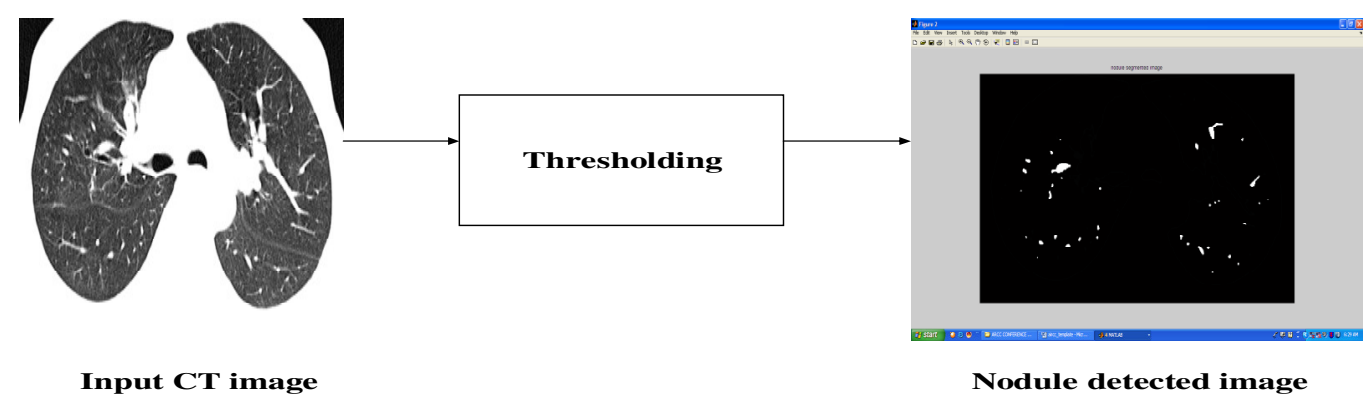

Figure 10.Nodule Segmented Image 


\section{RESULTS AND DISCUSSION}

\subsection{Fissure Verification}

In order to test the segmentation algorithm, real time clinical CT images from twenty patients have been processed and the experimental results are shown in Figure 11 and 12. The resolution of all CT images is $512 * 512$ pixels with a thickness of $5 \mathrm{~mm}$ to $10 \mathrm{~mm}$. The results were obtained for eight patients.

The performance of the segmentation algorithm is evaluated using a 1.6Ghz Intel(R) Core(TM) 2Duo CPU with 1GB of RAM running Matlab7.0 (R14). The Matlab is a Matrix laboratory and it is a high-performance language for technical computing integrates computation, visualization, and programming in an easy-to-use environment. Normally for surgeons it takes around 15 to 25 minutes to analyze the clinical CT images whereas the run time of segmentation algorithm is only 3 minutes and this indicates that the automatic segmentation is better suited for busy clinical settings.

The identified fissure results are verified by comparing the manual and the automatic segmentation and the accuracy is calculated statistically. Manual segmentation is carried out by the surgeons. In Figure 11, fissures are located manually by surgeons and the fissure distances are identified from two extreme boundaries. In Figure 12, oblique fissures are identified automatically by segmentation algorithm using fissure sweeping technique and its accuracy is found and it is shown in Table 2.

The automatic segmentation is better than manual segmentation because of the following reasons:

i. Automatic method is faster.

ii. Automatic method is easy to segment lung lobes with large number of images whereas it is difficult for manual method to segment lung lobes as the number of images is larger.

iii. Automatic method takes lesser time, whereas the manual method is clumsy and subjective.

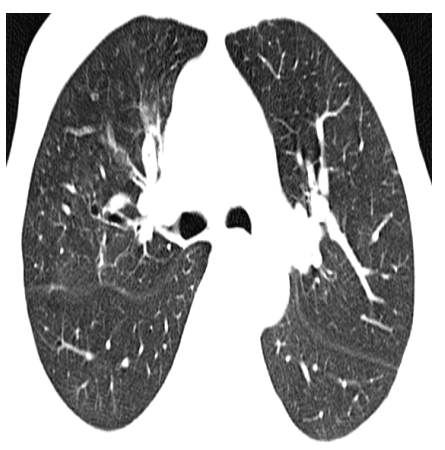

Input CT Image

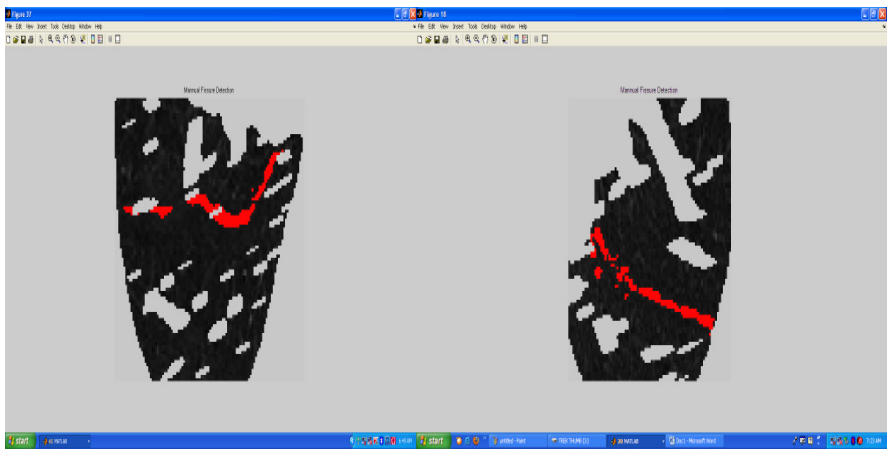

Manually segmented right and left oblique fissures

Figure11. Result for the Manual Segmentation 


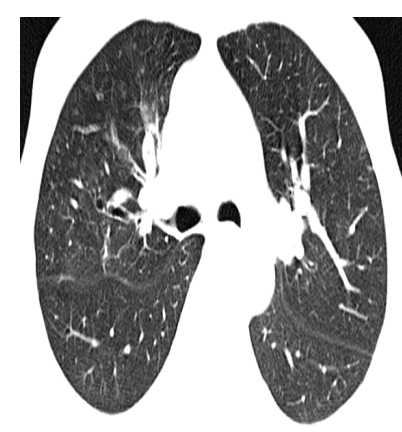

Input CT Image

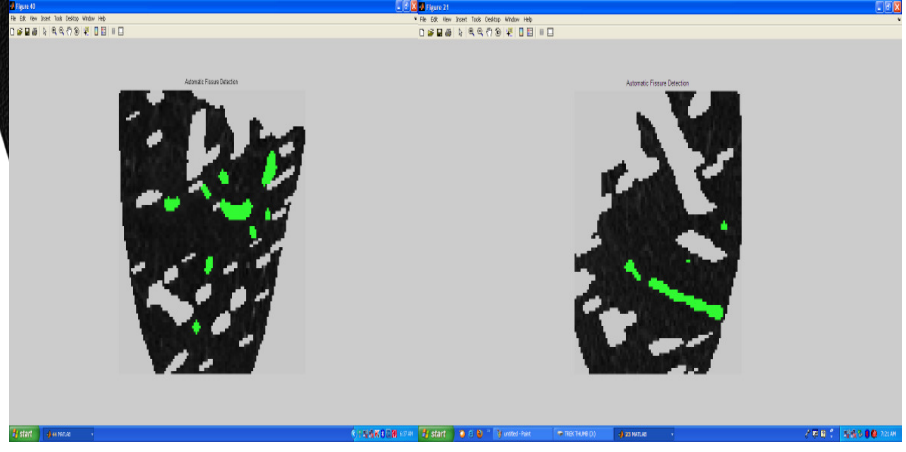

Automatically segmented right and left oblique fissures

Figure12. Result for the Automatic Segmentation

\begin{tabular}{|c|c|c|c|c|}
\hline Patient \# & $\begin{array}{c}\text { Manual } \\
\text { Segmentation } \\
\mathrm{M}_{\mathrm{a}}\end{array}$ & $\begin{array}{c}\text { Automatic } \\
\text { Segmentation } \\
\mathbf{A}_{\mathrm{a}}\end{array}$ & $\begin{array}{c}\text { Mean } \\
\text { Difference } \\
\mathbf{M}_{\mathbf{d}}=\mathbf{a b s}\left(\mathbf{M}_{\mathbf{a}}-\mathbf{A}_{\mathbf{a}}\right)\end{array}$ & $\begin{array}{c}\text { Accuracy } \% \\
\left(100 * M_{d}\right) /\left(M_{a}+A_{a}\right)\end{array}$ \\
\hline 1 & 220 & 161 & 59 & 87 \\
\hline 2 & 191 & 161 & 30 & 92 \\
\hline 3 & 1654 & 3661 & 2007 & 72.58 \\
\hline 4 & 1408 & 4263 & 2855 & 66.5 \\
\hline 5 & 639 & 3274 & 2635 & 60 \\
\hline 6 & 161 & 214 & 53 & 88 \\
\hline 7 & 84 & 85 & 31 & 82 \\
\hline 8 & 1270 & 4133 & 2863 & 65.3 \\
\hline
\end{tabular}

Table 2.Accuracy for Segmenting the Oblique Fissure

In Table 2, $\mathrm{M}_{\mathrm{a}}$ represents the total number of pixels from the edge to the centre of the fissure region in Manual Segmentation, $A_{a}$ represents the total number of pixels from the edge to the centre of the fissure region in Automatic Segmentation, $M_{d}$ represents the Mean difference between Manual and the Automatic Segmentation and finally accuracy is calculated using the following formula:

Accuracy $=\left(100 * \mathrm{M}_{\mathrm{d}}\right) /\left(\mathrm{M}_{\mathrm{a}}+\mathrm{A}_{\mathrm{a}}\right)$

\section{CONCLUSION AND FUTURE WORK}

Fissures cannot be seen by naked eyes because CT images are taken by low resolution scanners and as the number of image increases, efforts taken by the medical experts to analyze the image consumes much time. Because of this reason, there is a proposal for developing an automated segmentation system to assist the medical experts, thereby reducing the consumption of time, so the project has been developed.

In this project initially pre-processing of a CT image is done to remove the noises present in it. Then the vessels are enhanced using the morphological operators followed by lung segmentation. The fissure regions are identified, enhanced and verified. Lung nodules are segmented using 
adaptive threshold. Through the project we have developed a segmentation algorithm for identifying the fissures and nodules from CT images. Using the statistical analysis such as mean difference between the automatic and manual segmentation, the results are verified. The results indicate a potential for developing an automatic algorithm to segment lung lobes for surgical planning of treating lung disease.

This project may be extended in the future, by focussing on the following aspects: Improving segmentation of horizontal fissures, further evaluating the algorithm under severe pathological cases, 3D visualization of the segmented lung lobes and nodules using volume rendering method and fissure detection, verification is carried out with the increased number of images.

\section{REFERENCES}

[1] AubertG and KornprobstP.(2006),'Mathematical Problems in Image Processing:PartialDifferential Equations and the Calculus of Variations (AppliedMathematical Sciences 147).' New York: Springer-Verlag.

[2] Aykac, D. Hoffman E. A, McLennan G. and. Reinhardt J.M, (2003) 'Segmentationand analysis of the human airway tree from three-dimensional X-rayCT images', IEEE Trans. Med. Imag., vol. 22, no. 8, pp. 940-950, Aug.

[3] Graham M. W, Gibbs J. D., and. William E. HRobust( 2008 ), system forhuman airway-tree segmentation',Proc. SPIE (Med. Imag.), vol. 6914, pp. 69141J-1-69141J-18.

[4] Grady L. (2006), 'Random walks for image segmentation', IEEE Trans. Pattern Anal. Mach. Intell., vol. 28 , no. 11 , pp. 1768-1783.

[5] Haralick R. M,. Stenberg, S. R and Zhuang, X.(1987) ,'Image analysis using mathematical morphology',IEEE Trans. Pattern Anal. Mach. Intell.,vol. 9, no. 4, pp. 532-550.

[6] Hu S HoffmanE.A and. Reinhardt J.M, (2001), 'Automatic lung segmentation for accurate quantitation of volumetric X-ray CT images’, IEEE Trans.

[7] Hemminger B.M,. Molina, P. L. Egan T. M.Detterbeck, Muller, K. E.Coffrey, and Lee, J. K. (2005), 'Assessment of real-time 3D visualization for cardiothoracic diagnostic evaluation and surgery planningDigit. Imag., vol. 18, pp. 145-153.

[8] Kass M,.Witkin A and.Terzopoulos D.(1987), 'Snakes:Active contour models,'Int. J. Comput. Vis., vol. v1, pp. 321-331.

[9] Kubo M,. Niki N. Eguchi K,. Kaneko M, and. Kusumoto M. (2000) 'Extraction of pulmonary fissures from thin-section CT images using calculation of surface-curvatures and morphology filters', in Proc. IEEE Int. Conf. Imag.Process. pp. 637-640.

[10] Kubo M,. Kawata, Y. Niki, N Eguchi K., Ohmatsu H.,. Kakinuma R,Kaneko M.,. Kusumoto M,. Moriyama N,. Mori K, and. Nishiyama H.(2001)‘Automatic extraction of pulmonary fissures from multidetector-row CTimages’, in Proc. IEEE Int. Conf. Imag. Process. pp. 1091-1094.

[11] Kuhnigk J.-M. Hahn H. Hindennach M. Dicken V. Krass S, and Peitgen H.O.(2003) 'Lung lobe segmentation by anatomy-guided 3-D watershed transform', Proc. SPIE (Med. Imag.), vol. 5032, pp. 1482-1490,Med. Imag., vol. 20, no. 6, pp. 490-498.

[12] Kubo M.. Niki N, Nakagawa S., Eguchi K.,. Kaneko M, Moriyama N, Omatsu H, Kakinuma R and Yamaguchi.N. (1999), 'Extraction algorithm of Pulmonary fissures from thin-section CT images based on linear feature Detector method', IEEE Trans. Nucl. Sci., vol. 46, no. 6, pp. 2128-2133.

[13] Kuhnigk J.-M,. Dicken V,. Zidowitz S, Bornemann L.,. Kuemmerlen B,Krass, S Peitgen, H.-O. Yuval, . S. Fend, H.-H Rau, W. S. and. Achenbach, T(2005),'Newtools for computer assistance in thoracic CT. Part 1. Functional analysis of lungs, lung lobes, and bronchopulmonary segments', Radio-Graphics, vol. 25, pp. 525-536.

[14] Sonka, M. Hlavac V, and Boyle R.(, 2008.),' Image processing, Analysis, and Machine Vision', 3rd ed. Toronto, ON: Thomson Learning. 
[15] SluimerI.ProkopM and GinnekenB.V (2005), 'Toward automated segmentation of the pathological lung in CT’, (IEEE Trans. Med. Imag., vol. 24, no. 8, pp. 1025-1038.

[16] Tschirren J. Hoffman, E. A. McLennan G, and. Sonka M.(2005),'Intrathoracic airway trees: Segmentation and airway morphology analysis from lowdose CT scans',IEEE Trans. Med. Imag., vol. 24, no. 12, pp. 1529-1539.

[17] Ukil S. Sonka M, and Reinhardt F.C (2006) 'Automatic segmentation of pulmonary fissures in Xray CT image using anatomic guidance’, Proc SPIE (Med. Imag.), vol. 6144, pp. 213-223.

[18] WangJ, BetkeM and. Ko, J. P. (2006).'Pulmonary fissure segmentation on CT' Med. Imag. Anal., vol. 10, pp. 530-547.

[19] Wang J Betke M and. Ko J. P. (2002), 'Segmentation of pulmonary fissures on diagnostic CTPreliminary experience’ in Proc. Int. Conf. Diagn. Imag.Anal, pp. 107-112.

[20] Wei Q Hu Y. Macgrego J. H r, and Gelfand G. (2008), 'Segmentation of lung lobes in clinical CT images', Int. J. CARS, vol. 3, pp. 151-163.

[21] WuZ and. LeahyR (1999),'an optimal graphs theoretic approach to data clustering: Theory and its application to image segmentation', IEEE Trans.Pattern Anal. Mach. Intell., vol. 15, no. 11, pp. 1101-1113.

[22] Zhang .L. Hoffman E. A and. Reinhardt J. M. (2006) 'Atlas-driven lung lobe segmentation in volumetric X-ray CT images’, IEEE Trans. Med. Imag.,vol. 25, no. 1, pp. 1-16.

[23] Zhang, L. E. Hoffman A. and. Reinhardt J. M.(2001),'Lung lobe segmentation by graph search with 3D shape constraints' Proc. SPIE (Med. Imag.),vol. 4321, pp. 204-215.

[24] Wei, Q., Hu, Y., Gelfand, G., \& MacGregor, J.H.,"Segmentation of Lung Lobes in High resolution Isotropic CT images” IEEE Trans. Med. Imag., vol. 56, no. 5, pp. 1-11, may 2009.

Authors

[1] S. Anitha is pursuing her Post Graduation in Multimedia Technology, Her Area of interest include Image Processing, Multimedia and Mobile Networks.

[2] Dr. S. Sridhar is an Assistant Professor in Computer Science and Engineering Department at College of Engineering, Guindy, Chennai. His Research interest is in the area of Image Processing,

Pattern Recognition, Data Mining, Artificial Intelligence.
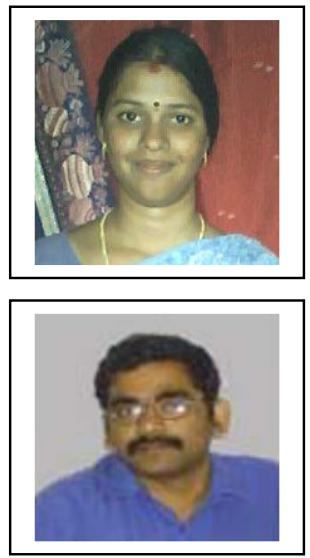